PROCEEDINGS OF THE

AMERICAN MATHEMATICAL SOCIETY

Volume 109, Number 4, August 1990

\title{
WEAKLY FACTORIAL DOMAINS AND GROUPS OF DIVISIBILITY
}

\author{
D. D. ANDERSON AND MUHAMMAD ZAFRULLAH
}

(Communicated by Louis J. Ratliff, Jr.)

\begin{abstract}
An integral domain $R$ is said to be weakly factorial if every nonunit of $R$ is a product of primary elements. We give several conditions equivalent to $R$ being weakly factorial. For example, we show that the following conditions are equivalent: (1) $R$ is weakly factorial; (2) every convex directed subgroup of the group of divisibility of $R$ is a cardinal summand; (3) if $P$ is a prime ideal of $R$ minimal over a proper principal ideal $(x)$, then $P$ has height one and $(x)_{P} \cap R$ is principal; (4) $R=\cap R_{P}$, where the intersection runs over the height-one primes of $R$, is locally finite, and the $t$-class group of $R$ is trivial.
\end{abstract}

Throughout this note, $R$ will be a commutative integral domain with identity having quotient field $K$. Suppose that $R$ is a UFD. If $S$ is a saturated multiplicatively closed subset of $R$, then $S=\left\{\lambda p_{1} \cdots p_{n} \mid \lambda\right.$ a unit of $R, n \geq 0$, each $\left.\left(p_{i}\right) \in Y\right\}$ for some subset $Y \subseteq X^{(1)}$, where $X^{(1)}$ is the set of height-one prime ideals of $R$. Conversely, every such subset $Y \subseteq X^{(1)}$ determines a saturated multiplicatively closed subset of $R$. If we set $T=\left\{\lambda p_{1} \cdots p_{n} \mid \lambda\right.$ a unit of $R, n \geq 0$, each $\left.\left(p_{i}\right) \in X^{(1)}-Y\right\}$, then every nonzero element $r$ of $R$ may be written uniquely up to units in the form $r=s t$ where $s \in S$ and $t \in T$. Stated in terms of the group of divisibility $G(R)$ of $R,\langle S\rangle$ is a cardinal summand of $G(R)$. In fact, $\langle S\rangle \oplus_{\mathrm{C}}\langle T\rangle=G(R)$. (See the next paragraph for definitions.) Here $G(R)$ is a cardinal sum of copies of $\mathbb{Z}$, one for each height-one prime, and $\langle S\rangle$ is the sum of the cyclic summands corresponding to the primes from $Y$. The purpose of this paper is to characterize the integral domains with this property. We show that an integral domain $R$ has the property that every convex directed subgroup of $G(R)$ is a cardinal summand of $G(R)$ if and only if every nonunit of $R$ is a product of primary elements, that is, $R$ is weakly factorial.

Let $R$ be an integral domain with quotient field $K$. The group of divisibility of $R$ is the group $G(R)=K^{*} / U(R)$, where $K^{*}$ is the multiplicative group of $K$ and $U(R)$ is the group units of $R, G(R)$ is partially ordered

Received by the editors July 1, 1989 and, in revised form, November 6, 1989.

1980 Mathematics Subject Classification (1985 Revision). Primary 13A05, 13A17, 13F99, $06 \mathrm{~F} 20$.

*This paper was written while the second author was visiting the University of Iowa 
by $x U(R) \leq y U(R) \Leftrightarrow x \mid y$ in $R$. For results on partially ordered abelian groups and groups of divisibility, the reader is referred to [6, Chapter $3 ; 10]$. If $S$ is a saturated multiplicatively closed subset of $R$, then it is easily seen that $\langle S\rangle=\left\{s_{1} s_{2}^{-1} U(R) \mid s_{1}, s_{2} \in S\right\}$ is a convex directed subgroup of $G(R)$. Conversely, Mott [10, Theorem 2.1] has shown that every convex directed subgroup of $G(R)$ has this form. A subgroup $H$ of $G(R)$ is called a cardinal summand of $G(R)$ if there is a convex directed subgroup $L$ of $G(R)$ with $G(R)=H \oplus_{\mathrm{C}} L$, that is, $G(R)$ is the algebraic direct sum of $H$ and $L$ and the partial order on $G(R)=H \oplus L$ is given by $\left(h_{1}, l_{1}\right) \leq\left(h_{2}, l_{2}\right) \Leftrightarrow h_{1} \leq h_{2}$ and $l_{1} \leq l_{2}$. Mott and Schexnayder [11, Proposition 4.1] have shown that a saturated multiplicatively closed subset $S$ of $R$ has $\langle S\rangle$ as a cardinal summand of $G(R)$ if and only if there is a saturated multiplicatively closed subset $T$ of $R$ with $S \cap T=U(R)$ such that every nonzero element $r$ of $R$ can be written uniquely up to units in the form $r=s t$, where $s \in S, t \in T$ and each element $s$ of $S$ is $v$-coprime to each element $t \in T$ in the sense that $(s, t)_{v}=R$ or, equivalently, that $(s) \cap(t)=(s t)$. Here $v$ denotes the $v$-operation, that is, for an ideal $I$ of $R, I_{v}=\left(I^{-1}\right)^{-1}=[R:[R: I]]$.

An element $x \in R$ is said to be primary if $(x)$ is a primary ideal of $R$ and an integral domain $R$ is said to be weakly factorial if every (nonzero) nonunit of $R$ is a finite product of primary elements. Weakly factorial domains were introduced in [2]. Examples of weakly factorial domains include UFD's, onedimensional semi-quasi-local domains, and GCD domains which are generalized Krull domains. It was shown that, for $R$ weakly factorial, $R=\bigcap_{P \in X^{(1)}} R_{P}$, where the intersection is locally finite. Also, a Krull domain is factorial if and only if it is weakly factorial. Hence, a weakly factorial Noetherian domain is factorial if and only if it is integrally closed.

For a Krull domain $R$, the intersection $R=\bigcap_{P \in X^{(1)}} R_{P}$ is locally finite, and $R$ is fractorial if and only if $C l(R)=0$ where $C l(R)$ is the divisor class group of $R$. Since the divisorial ideals form a group under the $v$-product $\left(A * B=(A B)_{v}\right)$ if and only if $R$ is completely integrally closed, $C l(R)$ need not be a group for an arbitrary integral domain. However, for any integral domain $R$, there is a group $C l_{t}(R)$ called the $t$-class group of $R$ which agrees with $C l(R)$ when $R$ is a Krull domain. A nonzero fractional ideal $I$ of $R$ is said to be $t$-invertible if there is a fractional ideal $B$ of $R$ with $(A B)_{t}=R$. Here $t$ denotes the $t$-operation given by $I_{t}=\cup\left\{\left(a_{1}, \ldots, a_{n}\right)_{v} \mid 0 \neq\left(a_{1}, \ldots, a_{n}\right) \subseteq I\right\}$. An ideal $I$ is said to be a $t$-ideal if $I_{t}=I$. The $t$-operation is an example of a finite-character star operation. For results on star operations, the reader is referred to [6, $\S \S 32$ and 34]. For any integral domain $R$, the set of $t$-invertible $t$-ideals forms a group under the $t$-product $A * B=(A B)_{t}$. The $t$-class group of $R$ is $C l_{t}(R)$ - the group of $t$-invertible $t$-ideals modulo the subgroup of principal fractional ideals. For results on the $t$-class group, the reader is referred to $[4,5]$. We show that, for a domain $R, R$ is weakly factorial if and only if $R=\bigcap_{P \in X^{(1)}} R_{P}$ is locally finite and $C l_{t}(R)=0$. This result is part of the next theorem, the main result of this paper. 
Theorem. Let $R$ be an integral domain with quotient field $K$. Then the following statements are equivalent:

(1) Every convex directed subgroup of $G(R)$ is a cardinal summand of $G(R)$.

(2) For each saturated multiplicatively closed subset $S$ of $R,\langle S\rangle$ is a cardinal summand of $G(R)$.

(3) For each prime ideal $P$ of $R,\langle R-P\rangle$ is a cardinal summand of $G(R)$.

(4) $R$ is weakly factorial, that is, every nonunit of $R$ is a product of primary elements.

(5) $R=\cap_{P \in X^{(1)}} R_{P}$ where the intersection is locally finite and the natural map $G(R) \rightarrow \oplus_{P \in X^{(1)}} G\left(R_{P}\right)$ is surjective (and hence an order isomorphism).

(6) If $P$ is a prime ideal of $R$ minimal over a proper principal ideal $(x)$, then $h t P=1$ and $(x)_{P} \cap R$ is principal.

(7) $R=\bigcap_{P \in X^{(1)}} R_{P}$, where the intersection is locally finite and $C l_{t}(R)=0$.

Proof. (1) $\Leftrightarrow(2)$. This follows from the previously mentioned result of Mott [10, Theorem 2.1] that every convex directed subgroup of $G(R)$ has the form $\langle S\rangle$ for some saturated multiplicatively closed subset $S$ of $R$, and conversely. $(2) \Rightarrow(3)$. Clear. $(3) \Rightarrow(6)$. Let $P$ be a prime ideal minimal over $(x)$. Then $P$ is a $t$-ideal. Suppose that there is a prime ideal $Q$ with $0 \neq Q \subsetneq P$. Let $S=R-Q$. Then $\langle S\rangle$ is a cardinal summand of $G(R)$. By [11, Proposition 4.1] there is a multiplicatively closed subset $T$ of $R$ with $S \cap T=U(R)$, $S T=R-\{0\}$, and, for $s \in S$, and $t \in T,(s, t)_{v}=R$. Now $P \cap S \neq \varnothing$, so there exists an $s_{0} \in P \cap S$. Let $0 \neq q \in Q$, so $q=s t$ where $s \in S$ and $t \in T$. Then $s \notin Q$, so $t \in Q$. Thus $s_{0}, t \in P$ and hence $R=\left(s_{0}, t\right)_{v} \subset$ $P_{t}=P$, a contradiction. Therefore, $h t P=1$. (This shows that, in a weakly factorial domain, every prime $t$-ideal has height one.) It follows from [11, Proposition 4.1], taking $S=R-P$, that $(x)_{P} \cap R$ is principal. (6) $\Rightarrow(4)$. Let $S$ be the multiplicatively closed subset of $R$ consisting of all products of primary elements and units. We first show that $S$ is saturated. Let $x \in S$ be a nonunit and $y$ be a nonunit factor of $x$. If $x=q_{1} \cdots q_{r}$, where $\left(q_{i}\right)$ is $P_{i}$-primary, then $\left\{P_{1}, \ldots, P_{r}\right\}$ is the set of height-one primes containing $(x)$. Hence the set of height-one primes containing $(y)$ is a subset of $\left\{P_{1}, \ldots, P_{r}\right\}$, say $\left\{P_{1}, \ldots, P_{n}\right\}$. Now $(y)_{P_{1}} \cap R$ is principal, say $(y)_{P_{1}} \cap R=\left(q_{1}^{\prime}\right)$. Since $\operatorname{dim} R_{P_{1}}=1,\left(q_{1}^{\prime}\right)$ is $P_{1}$-primary. Now $y \in\left(q_{1}^{\prime}\right)$, so $y=q_{1}^{\prime} t_{1}$ where $t_{1} \notin P_{1}$, but $t_{1} \in P_{2} \cap \cdots \cap P_{n}$. Continuing in this manner, we get that $y=q_{1}^{\prime} \cdots q_{n}^{\prime} t_{n}$, where $\left(q_{i}^{\prime}\right)$ is $P_{i}$-primary and $t_{n}$ is contained in no height-one prime ideal, hence a unit. Suppose that some nonunit $a \in R$ is not in $S$. Then $(a) \cap S=\phi$ since $S$ is saturated. Hence $(a)$ can be enlarged to a prime ideal $P \supseteq(a)$ maximal with respect to missing $S$. Shrinking $P$ to a prime ideal $Q$ minimal over $(a)$, we have a height-one prime ideal $Q$ containing no primary elements. But this is absurd, for the ideal $(a)_{Q} \cap R$ is itself a principal primary ideal contained in $Q$. (4) $\Rightarrow(2)$. Let $R$ be a weakly factorial integral domain and let $S$ be a saturated multiplicatively closed subset of $R$. Let $x \in S$ be a 
nonunit and suppose that $x=q_{1} \cdots q_{n}$ where $\left(q_{i}\right)$ is $P_{i}$-primary. Let $(q)$ be $P_{i}$-primary. Then, for some $s \geq 1, q_{i}^{s} \in(q)$, so $q_{i}^{s}=r q$ for some $r \in R$; hence $q$ is a factor of $x^{s} \in S$. Since $S$ is saturated, $q \in S$. Thus it is easily seen that $S=\left\{\lambda q_{1} \cdots q_{n} \mid \lambda \in U(R), n \geq 0\right.$, each $\left(q_{i}\right)$ is $P_{i}$-primary where $P_{i} \in X^{(1)}$ with $\left.P_{i} \cap S \neq \varnothing\right\}$. Let $T=\left\{\lambda q_{1} \cdots q_{n} \mid \lambda \in U(R), n \geq 0\right.$, $\left(q_{i}\right)$ is $P_{i}$-primary where $\left.P_{i} \cap S=\varnothing\right\}$. Then $T$ is a saturated multiplicatively closed subset of $R$ satisfying the conditions of part (2) of [11, Proposition 4.1]. Hence by this proposition, $\langle S\rangle$ is a cardinal summand of $G(R) .(4) \Rightarrow(5)$. By [2, Corollary 14], $R=\bigcap_{P \in X^{(1)}} R_{P}$ and this intersection is locally finite. Let $0 \neq t \in R_{P}$ be a nonunit where $P \in X^{(1)}$. Then $t=q u$ where $(q)$ is $P$ primary and $u$ is a unit of $R_{P}$. Thus $q U(R) \rightarrow t U\left(R_{P}\right)$. It easily follows that the natural map $G(R) \rightarrow \bigoplus_{P \in X^{(1)}} G\left(R_{P}\right)$ is surjective and hence is an order isomorphism. $(5) \Rightarrow(4)$. Let $0 \neq r \in R$ be a nonunit. Let $P_{1}, \ldots, P_{n}$ be the height-one prime ideals containing $(r)$. Now, since the natural map $G(R) \rightarrow$ $\bigoplus_{P \in X^{(1)}} G\left(R_{P}\right)$ is surjective, for each $i=1, \ldots, n$, there exists a $k_{i} \in K$ with $k_{i} U\left(R_{P_{i}}\right)=r U\left(R_{P_{i}}\right)$ and $k_{i} U\left(R_{P}\right)=1 U\left(R_{P}\right)$ for each $P \in X^{(1)}-\left\{P_{i}\right\}$. Note that each $k_{i} \in \bigcap_{P \in X^{(1)}} R_{P}=R$. Moreover, since $P_{i}$ is the only height-one prime ideal containing $\left(k_{i}\right),\left(k_{i}\right)=\bigcap_{P \in X^{(1)}}\left(k_{i} R_{P}\right)=k_{i} R_{P_{i}} \cap R$ is $P_{i}$-primary. Now $r U\left(R_{P}\right)=k_{1} \cdots k_{n} U\left(R_{P}\right)$ for each $P \in X^{(1)}$, so $r=u k_{1} \cdots k_{n}$, where $u$ is a unit of $R$. (4) $\Rightarrow$ (7). By [2, Corollary 14], $R=\bigcap_{P \in X^{(1)}} R_{P}$, where the intersection is locally finite. Let $A$ be a $t$-invertible ideal of $R$. We must show that $A_{t}$ is principal. We may assume that $A$ is an integral ideal. We have already observed that every maximal $t$-ideal of $R$ has height one. Hence, by a result of Griffin [7, Proposition 4], $A_{P}$ is principal for each $P \in X^{(1)}$. Moreover, since the intersection is locally finite, $A_{P}=R_{P}$ except, say, for $P_{1}, \ldots, P_{n} \in X^{(1)}$. But at $P_{i}, A_{P_{i}}=q_{i} R_{P_{i}}$, where $\left(q_{i}\right)$ is $P_{i}$-primary. So $A \subseteq\left(q_{i}\right)_{P_{i}} \cap R=\left(q_{i}\right)$ and hence $A \subseteq\left(q_{1}\right) \cap \cdots \cap\left(q_{n}\right)=\left(q_{1} \cdots q_{n}\right)$. Thus $A=q_{1} \cdots q_{n} A^{\prime}$, where $A^{\prime}$ is contained in no height-one prime ideals. Hence $A_{t}^{\prime}=R$. So $A_{t}=\left(q_{1} \cdots q_{n} A^{\prime}\right)_{t}=\left(q_{1} \cdots q_{n} A_{t}^{\prime}\right)_{t}=\left(q_{1} \cdots q_{n}\right)_{t}=\left(q_{1} \cdots q_{n}\right)$ is principal. (7) $\Rightarrow(4)$. Let $0 \neq x \in R$ be a nonunit. Let $P_{1}, \ldots, P_{n}$ be the height-one prime ideals containing $(x)$. Let $Q_{i}=(x)_{P_{i}} \cap R$, so $(x)=$ $Q_{1} \cap \cdots \cap Q_{n}$, where $Q_{i}$ is $P_{i}$-primary. Now $x^{-1} Q_{1} \cdots Q_{n}$ is an ideal of $R$ and $x^{-1} Q_{1} \cdots Q_{n} R_{P}=R_{P}$ for each height-one prime ideal of $R$. Hence, by [1, Theorem 1], $\left(x^{-1} Q_{1} \cdots Q_{n}\right)^{*}=R$ where $*$ is the finite character star operation given by $A^{*}=\bigcap_{P \in X^{(1)}} A R_{P}$. Since * has finite character, $R=$ $\left(x^{-1} Q_{1} \cdots Q_{n}\right)^{*} \subseteq\left(x^{-1} Q_{1} \cdots Q_{n}\right)_{l} \subseteq R$, so $\left(x^{-1} Q_{1} \cdots Q_{n}\right)_{t}=R$. Thus each $Q_{i}$ is $t$-invertible and hence $Q_{i t}=\left(q_{i}\right)$ for some $q_{i} \in R$. Also, $(x)=\left(Q_{1} \cdots Q_{n}\right)_{t}=$ $\left.\left(Q_{1 t} \cdots Q_{n t}\right)_{t}=\left(\left(q_{1}\right)\right) \cdots\left(q_{n}\right)\right)_{t}=\left(q_{1}\right) \cdots\left(q_{n}\right)$. Now $\left(q_{i}\right) \supseteq Q_{i}$ and hence $\left(q_{i}\right)_{P}=$ $R_{P}$ except possibly at $P=P_{i}$. So $Q_{i}=(x)_{P_{i}} \cap R=\left(q_{i}\right)_{P_{i}} \cap R=q_{i} R_{P_{i}} \cap$ $\left(\bigcap_{P \in X^{(1)}-\left\{P_{i}\right\}} q_{i} R_{P}\right)=\left(q_{i}\right)$. Hence $\left(q_{i}\right)$ is $P_{i}$-primary, so $x$ is a product of primary elements. 
Corollary 1. Let $R$ be a Noetherian integral domain. Then $R$ is weakly factorial if and only if every grade-one prime ideal has height one and $C l_{t}(R)=0$.

Proof. $(\Rightarrow)$. A grade-one prime ideal is contained in a maximal $t$-ideal and hence has height one. By the previous theorem, $C l_{t}(R)=0$. $(\Leftarrow)$. If every grade-one prime ideal has height one, $R=\bigcap_{P \in X^{(1)}} R_{P}$, where the intersection is locally finite [8, Theorem 53]. By the previous theorem, $R$ is weakly factorial.

In the spirit of Mott [9], we use the Theorem and the Krull-KaplanskyJaffard-Ohn Theorem to obtain a result of A. Bigard (see, for example, [3, Theoreme 14.4.3, pp. 290-291]) concerning lattice ordered groups.

Corollary 2 (A. Bigard). A lattice ordered abelian group $G$ is a cardinal sum of Archimedian totally ordered groups if and only if every convex directed subgroup of $G$ is a cardinal summand of $G$.

Proof. Let $G$ be a lattice ordered abelian group. By the Krull-KaplanskyJaffard-Ohm Theorem ([6, Theorem 18.6] or [9, Theorem 2.1]), $G$ is order isomorphic to the group of divisibility of a Bézout domain $R$. By the Theorem, $G(R)$, and hence $G$, has the property that every convex directed subgroup is a cardinal summand if and only if $R$ is weakly factorial. But by [2, Theorem 21] a Bézout domain $R$ is weakly factorial if and only if $G(R)$ is order isomorphic to a cardinal sum of rank one (equivalently, Archimedian) totally ordered abelian groups.

As previously stated, examples of weakly factorial domains include UFD's and one-dimensional semi-quasi-local domains. Actually, it is easily seen that a one-dimensional domain $R$ is weakly factorial if and only if $R$ is Laskerian (i.e., every ideal has a primary decomposition) and $\operatorname{Pic}(R)=0$. Also, an integral domain that is both a GCD-domain and a generalized Krull domain is weakly factorial. (An integral domain $R$ is called a generalized Krull domain if $R=\cap V_{\lambda}$, where each $V_{\lambda}$ is an essential rank one valuation overring of $R$ and the intersection is locally finite.) In fact, according to [2, Theorem 20], the following conditions on an integral domain $R$ are equivalent: (1) $R$ is a weakly factorial GCD-domain; (2) $R$ is weakly factorial generalized Krull domain; and (3) $R$ is a generalized Krull domain and a GCD-domain. To this list may be added (4) $R$ is weakly factorial and, for each height-one prime ideal $P$ of $R$ (equivalently, maximal $t$-ideal $P$ of $R$ ), $R_{P}$ is a valuation domain and (5) $R$ is weakly factorial and if $p$ and $q$ are non $v$-coprime primary elements of $R$, then $p \mid q$ or $q \mid p$. (Use [2, Theorem 18] and its proof). We next give another source of weakly factorial domains.

Example. Let $K \subseteq L$ be fields. Then $R=K+X L[X]=\{f(X) \in L[X] \mid f(0) \in$ $K\}$ is an atomic weakly factorial domain. It is easily seen that every nonzero nonunit of $R$ may be written uniquely in one of the following two forms: $p_{1} \cdots p_{n}$ or $p_{1} \cdots p_{n}\left(a X^{r}\right)$, where $p_{1}, \ldots, p_{n}$ are principal primes of $R$ and $a X^{r}(r \geq 1, a \in L)$ is primary. 
It is well known [8, Theorem 5] that an integral domain $R$ is a UFD if and only if every nonzero prime ideal contains a nonzero principal prime ideal. The proof is based on the fact that the set of principal primes forms a saturated multiplicatively closed set. A similar result for weakly factorial domains involving primary elements is not true.

For let $R$ be a Dedekind domain that is not a PID, but has torsion class group. Since some power of each prime ideal is principal, each prime ideal contains a principal primary ideal, but $R$ is not weakly factorial. In this case, the multiplicatively closed set of all products of primary elements is not saturated. As a concrete example, suppose that $R$ has nonprincipal maximal ideals $M$ and $N$ with $M^{2}=(x), N^{2}=(y)$, and $M N=(z)$. Then $\left(z^{2}\right)$ $=(M N)^{2}=M^{2} N^{2}=(x y)$. So $z^{2}$ is a product of primary elements, but $z$ itself is not. Note that $x, y$, and $z$ are all irreducible. Thus a single irreducible element of a Krull domain may be primary without being prime. However, a Krull domain is a UFD if and only if each irreducible element is primary.

However, for any integral domain $R$ and any nonzero prime ideal $P$ of $R$, it is easily proved that $S_{P}=\left\{\lambda p_{1} \cdots p_{n} \mid \lambda \in U(R), n \geq 0,\left(p_{i}\right)\right.$ is $P$-primary $\}$ is a saturated multiplicatively closed subset of $R$. Let $S$ be the multiplicatively closed subset of $R$ consisting of all products of primary elements. Then, in $G(R),\langle S\rangle=\oplus\left\langle S_{P}\right\rangle$ where the direct sum runs over all nonzero primes $P$ of $R$ with $P$ containing a $P$-primary element. Even though each $\left\langle S_{P}\right\rangle$ is a convex directed subgroup of $G(R), \oplus\left\langle S_{P}\right\rangle$ need not be convex, in fact, $\oplus\left\langle S_{P}\right\rangle$ is convex if and only if $S$ is saturated. Returning to the case where $R$ is a Dedekind domain with torsion class group but is not a PID, we see that each $\left\langle S_{P}\right\rangle$ is order isomorphic to $(\mathbb{Z},+)$. It is interesting to note that in this case we have $\bigoplus_{P \in X^{(1)}}\left\langle S_{P}\right\rangle \subsetneq G(R) \subsetneq \bigoplus_{P \in X^{(1)}} G\left(R_{P}\right)$, with $\bigoplus_{P \in X^{(1)}}\left\langle S_{P}\right\rangle$ being a directed lattice ordered subgroup of $G(R)$ and $G(R)$ being a directed lattice ordered subgroup of $\bigoplus_{P \in X^{(1)}} G\left(R_{P}\right)$. Moreover, all three groups are (algebraically) isomorphic to a direct sum of $\left|X^{(1)}\right|$ copies of $(\mathbb{Z},+$ ).

\section{REFERENCES}

1. D. D. Anderson, Star-operations induced by overrings, Comm. Alg. 16 (1988), 2535-2553.

2. D. D. Anderson and L. A. Mahaney, On primary factorizations, J. Pure Appl. Alg. 54 (1988), 141-154.

3. A. Bigard, K. Keimal, and S. Wolfenstein, Groupes et anneaux réticulés, Lecture Notes in Math., vol. 608, Springer-Verlag, New York, 1977.

4. A. Bouvier, Le groupe des classes d'un anneau integré, $107^{e}$ Congrés national des Sociétés savantes, Brest, 1982, sciences, fasc, IV, 85-92.

5. A. Bouvier and M. Zafrullah, On some class groups of an integral domain, Bull. Soc. Math. Grèce (to appear).

6. R. Gilmer, Multiplicative ideal theory, Marcel Dekker, New York, 1972.

7. M. Griffin, Some results on v-multiplication rings, Canad. J. Math. 19 (1967), 710-722.

8. I. Kaplansky, Commutative rings, revised ed., The University of Chicago Press, Chicago and London, 1974. 
9. J. L. Mott, The group of divisibility and its application, Lecture Notes in Math., vol. 311, Springer-Verlag, New York, 1972, 194-208.

10. $\ldots$, Convex directed subgroups of a group of divisibility, Canad. J. Math. 26 (1974), 532542.

11. J. L. Mott and M. Schexnayder, Exact sequences of semi-value groups, J. Reine Angew. Math. 283/284 (1976), 388-401.

Department of Mathematics, The University of Iowa, Iowa City, Iowa 52242

Department of Mathematics, Winthrop College, Rock Hill, South Carolina 29733 